\title{
$4 \quad$ Umgang mit autismusspezifischen herausfordernden Verhaltensweisen
}

\author{
Bettina Brehm, Judith E. Schill, Monica Biscaldi, Christian Fleischhaker
}

In den letzten Jahren sind die hochfunktionalen Autismus-Spektrum-Störungen (ASS) ins Zentrum der Aufmerksamkeit gerückt.

Die hochfunktionalen Kinder mit ASS haben nach § 35 a KJHG das Recht auf Eingliederungshilfe, d. h. zum Beispiel auch einen Anspruch auf einen Schulbegleiter im Sinne der Inklusion. Viele Kinder mit ASS besuchen derzeit somit immer häufiger eine Regelschule mit einem Schulbegleiter. Daraus sind zahlreiche Fragen im Spannungsfeld Lehrer, Eltern und Schulbegleiterinnen entstanden.

Immer wieder treten Fragen auf, wie mit den Besonderheiten der Kinder im schulischen oder häuslichen Alltag umgegangen werden kann. Aus diesem Bedarf heraus sind in den letzten Jahren zahlreiche Eltern- und Schulratgeber entstanden (Richman 2004; Schirmer 2010; Schuster 2011; Schuster \& Matzies-Köhler 2011; Schirmer 2006; Bernard-Opitz 2007b).

Es ergeben sich im Alltag für die Eltern und Lehrer insbesondere immer wieder Fragestellungen zum Umgang mit herausfordernden Verhaltensweisen wie Eskalationen, wiederkehrende kritische Situationen und Fragen zum Umgang mit stereotypen und ritualisierten Verhaltensweisen.

Beim Umgang mit Eskalationen schlagen Remschmidt \& Kamp-Becker (2006) vor, zunächst die Situation zu beenden, das Kind sich beruhigen zu lassen und danach eine Nachbesprechung durchzuführen.

In den Materialien für Lehrer und Schulbegleiter, herausgegeben von Autismus Deutschland e.V. (Thomas et al. 2005; Wilczek 2013), werden weiterhin Vorschläge zum Umgang mit mangelnder Flexibilität und besonderen Problembereichen wie Freundschaften, Stimmungsumschwüngen, flukturierender Aufmerksamkeit sowie wortwörtliches Verstehen gemacht, die im vorliegenden Manual immer wieder aufgegriffen werden.

Im Umgang mit Stereotypien stellt die Verhaltensanalyse bzw. systematische Verhaltensbeobachtung eine wichtige Voraussetzung zur Bestimmung der Funktion eines Verhaltens dar. Je nach Verhaltensanalyse kann zum einen die auslösende Situation verändert (Prävention, z. B. durch Reizabschirmung) oder es können zum anderen alternative Verhaltensweisen verstärkt und mit Hilfestellungen (Prompting) angelernt werden.

Eine ungewollte Verstärkung von stereotypem Verhalten im Alltag sollte vermieden und angemessenes Verhalten gezielt positiv verstärkt werden.

Durch das Fördern von sozial-kommunikativen Fähigkeiten (Wünsche äußern, um Hilfe bitten, Emotionen ausdrücken) und durch eine angemessene Alltagsstrukturierung soll weiterhin unangemessenes und stereotypes Verhalten reduziert werden. 